\title{
A half-a-day long thermonuclear X-ray burst from KS 1731-260
}

\author{
E. Kuulkers ${ }^{1,2}$, J. J. M. in 't Zand ${ }^{2,1}$, M. H. van Kerkwijk², R. Cornelisse',2, D. A. Smith ${ }^{3}$, J. Heise ${ }^{1}$, \\ A. Bazzano ${ }^{4}$, M. Cocchi ${ }^{4}$, L. Natalucci ${ }^{4}$, and P. Ubertini ${ }^{4}$ \\ 1 SRON National Institute for Space Research, Sorbonnelaan 2, 3584 CA Utrecht, The Netherlands \\ 2 Astronomical Institute, Utrecht University, PO Box 80000, 3508 TA Utrecht, The Netherlands \\ 3 University of Michigan, Department of Physics, Ann Arbor, MI 48109, USA \\ 4 Istituto di Astrofisica Spaziale (CNR), Area Ricerca Roma Tor Vergata, via del Fosso del Cavaliere, \\ 00133 Roma, Italy
}

Received 4 September 2001 / Accepted 6 November 2001

\begin{abstract}
We report on an approximately twelve hour long X-ray flare from the low-mass X-ray binary KS 1731-260. The flare has a rise time of less than $13 \mathrm{~min}$ and declines exponentially with a decay time of 2.7 hours. The flare emission is well described by black-body radiation with peak temperature of $2.4 \mathrm{keV}$. The total energy release from the event is $10^{42} \mathrm{erg}$ (for an assumed distance of $7 \mathrm{kpc}$ ). The flare has all the characteristics of thermo-nuclear X-ray bursts (so-called type I X-ray bursts), except for its very long duration and therefore large energy release (factor of 1500-4000 longer and 250-425 more energy than normal type I X-ray bursts from this source). The flare is preceded by a short and weak X-ray burst, possibly of type I. Days to weeks before the flare, type I X-ray bursts were seen at a rate of $\sim 3$ per day. However, after the flare type I X-ray bursting ceased for at least a month, suggesting that the X-ray flare affected the type I bursting behaviour. The persistent emission is not significantly different during the non-bursting period. We compare the characteristics of this event with similar long X-ray flares, so-called superbursts, seen in other sources (4U 1735-44, 4U 1820-30, $4 \mathrm{U}$ 1636-53, Ser X-1, GX 3+1). The event seen from KS 1731-260 is the longest reported so far. We discuss two possible mechanisms that might cause these superbursts, unstable carbon burning (as proposed recently) and electron capture by protons with subsequent capture of the resulting neutrons by heavy nuclei.
\end{abstract}

Key words. accretion, accretion disks - binaries: close - stars: individual (KS 1731-260) - stars: neutron X-rays: bursts

\section{Introduction}

Many low-mass X-ray binaries show thermo-nuclear explosions, or type I X-ray bursts (for a review see Lewin et al. 1993). These appear as rapid $(\sim 1 \mathrm{~s})$ increases in the $\mathrm{X}$-ray flux, followed by an exponential decline, with typical durations of the order of seconds to minutes. The (net) burst spectra are well described by black-body emission from a compact object with $\sim 10 \mathrm{~km}$ radius and temperature of $\sim 1-2 \mathrm{keV}$. The inferred temperature decreases during the decay, indicating cooling of the neutron star surface. Typical integrated burst energies are in the $\sim 10^{39}$ to $10^{40}$ erg range.

Recently, new modes of unstable burning on neutron stars may have been revealed, with the discovery of X-ray flares lasting several hours (Cornelisse et al. 2000; Strohmayer 2000; Strohmayer \& Brown 2001; Wijnands 2001; Kuulkers 2001a; Cornelisse et al. 2001). The recurrence times of these events are not well constrained.

Send offprint requests to: E. Kuulkers,

e-mail: E.Kuulkers@sron.nl
Wijnands (2001) reported two events from 4U 1636-53 which were $\simeq 4.7$ years apart. So far, only seven events have been found, indicating that they are rare. The availability of X-ray instruments such as the BeppoSAX/WFC and the RXTE/ASM, which more frequently monitor the $\mathrm{X}$-ray sky, and the RXTE/PCA, which performs long studies of the X-ray burster population, is the reason that these events have now been discovered.

These long X-ray flares share many of the characteristics of type I bursts, which is the reason they are attributed to thermonuclear runaway events (see e.g. Cornelisse et al. 2000). What distinguishes them from normal type I bursts are the long duration (exponential decay times of a few hours), the large fluences $\left(\sim 10^{42} \mathrm{erg}\right)$, and the aforementioned extreme rarity. Because of the large fluences, they are also referred to as "superbursts" So far, these events have only been observed in sources with persistent pre-burst luminosities of $\sim 0.1-0.3$ times the Eddington luminosity (e.g. Wijnands 2001).

The current view is that the superbursts are caused by the unstable burning of the ashes of the (un)stable 
hydrogen and/or helium burning (Cumming \& Bildsten 2001; Strohmayer \& Brown 2001). Such bursts in principle thus not only tell us about properties of material buried below the hydrogen and/or helium layer, but also about the burning of the hydrogen and/or helium layer itself.

We here report on a superburst seen with the BeppoSAX Wide Field Camera (WFC) from KS 1731-260 on 1996 September 23. In Sect. 2, we briefly review what is known about KS 1731-260. Next, we describe the observations (Sect. 3) and the results (Sect. 4). We conclude with a discussion of possible mechanisms for the superbursts. Hereafter we use the term (X-ray) bursts, whenever we refer to type I (X-ray) bursts (unless it is needed for clarity).

\section{KS $1731-260$}

KS 1731-260 was discovered as a transient and bursting source in 1989 by COMIS/TTM onboard Mir-Kvant (Sunyaev et al. 1989, 1990). A possible infrared counterpart was identified recently using a precise localisation by Chandra (Wijnands et al. 2001b; Revnivtsev \& Sunyaev 2002; Groot et al. 2001, in preparation). The source is an X-ray burster (Sunyaev et al. 1990; Muno et al. 2000), which identifies the compact object as a neutron star. Highly coherent X-ray oscillations were discovered by the RXTE/PCA at a period of $1.9 \mathrm{~ms}$ during several X-ray bursts (Smith et al. 1997; Muno et al. 2000) as well as $\mathrm{kHz}$ quasi-periodic oscillations at $\sim 900 \mathrm{~Hz}$ and $\sim 1200 \mathrm{~Hz}$ in the persistent emission (Wijnands \& van der Klis 1997). The distance to the source, as derived from the peak luminosity observed in radius-expansion bursts, is about $7 \mathrm{kpc}$ (Muno et al. 2000).

Figure 1a shows the RXTE/ASM light curve of KS 1731-260 from near the start of the RXTE mission, i.e. 1996 Jan. 6, to 2001 May 1. The source is highly variable on time scales of a day to weeks. During the first two and a half year of the RXTE mission the overall source intensity varied slowly on time scales longer than a week. Subsequently, it showed a two and a half year interval with strong flares or outbursts on time scales of weeks to a month, reflecting a change in source state (see also Muno et al. 2000; Revnivtsev \& Sunyaev 2001). At the beginning of 2001, the source became quiescent (see Wijnands et al. 2001a).

\section{Observations}

BeppoSAX was launched on 1996 April 30. Onboard are two Wide Field Camera's (WFCs); they consist of coded mask aperture cameras (Jager et al. 1997). They point in opposite directions of one another and perpendicular to the Narrow-Field Instruments on the same satellite (Boella et al. 1997). The field of view of each WFC is $40 \times 40$ square degrees full-width to zero response with an angular resolution of about 5 arcmin in each direction. The band pass of the instruments is 2 to $28 \mathrm{keV}$.
The imaging capability and sensitivity allow an accurate monitoring of complex sky regions, like the Galactic Centre. Two times per year, in spring and autumn, for about two months a dedicated program is carried out to monitor the Galactic Centre region typically for about half a day on a weekly basis. During the performance verification phase, however, the Galactic Centre was monitored continuously (only interrupted by South Atlantic Anomaly passages and earth occultations) for an interval of $\sim 9$ days, four months after launch (see e.g. Cornelisse et al. 2000; Kuulkers et al. 2000). KS 1731-260 lies close to the Galactic Centre $\left(l=1.07^{\circ}, b=+3.66^{\circ}\right)$, ensuring the source is well covered. In this paper we use the data from either WFC unit 1 or 2, from the Galactic Centre monitoring program as well as from serendipitous observations close to the Galactic Centre, obtained in 1996 (net observing time of $851 \mathrm{ksec}), 1997$ (785 ksec) and northern spring 1998 (414 ksec). Data covering (part of) the Galactic Centre are routinely checked for X-ray bursts. We found a total of 63 bursts from KS 1731-260 in the above interval.

The Rossi X-ray Timing Explorer (RXTE) was launched 1995 December 30. This satellite carries three instruments, including the All-Sky Monitor (ASM), which consists of three Scanning Shadow Cameras (SSCs) mounted on a rotating drive such that one is perpendicular to the other two (Levine et al. 1996). The cameras are held stationary for 90-s intervals, called "dwells", during which data are accumulated. The mount rotates the assembly through a $6^{\circ}$ angle between dwells until a full rewind is necessary. In this manner, $\sim 80 \%$ of the sky is observed every 90-min orbit. Each dwell provides intensities in the $1.5-12 \mathrm{keV}$ band for all known sources in the field of view of each camera, and results that meet a set of reliability criteria are saved in electronic tables that are available over the world wide web ${ }^{1}$. An alternate, timeseries, data mode records the total counts in each SSC in 0.125 -s bins, but does not preserve the position of each photon (Levine et al. 1996; Smith et al. 2001). Over the $\sim 5.5$ years of RXTE operation, KS 1731-260 was observed an average of $\sim 10$ times a day, yielding $\sim 17000$ individual intensity measurements.

\section{Analysis and results}

\subsection{A long $X$-ray flare}

Following the discovery of the long X-ray flare in 4U 1735-44 (Cornelisse et al. 2000), we found two other such events in our WFC database of X-ray bursters: one from Ser X-1, presented by Cornelisse et al. (2001), the other from KS 1731-260, presented here. The long X-ray flare of KS 1731-260 can be seen clearly in Fig. 1b, which shows the WFC data obtained on KS 1731-260

\footnotetext{
1 These quick-look results can be found at http://xte.mit.edu, and final ASM data products can be found at http://heasarc.gsfc.nasa.gov/docs/xte/ asm_products.html.
} 

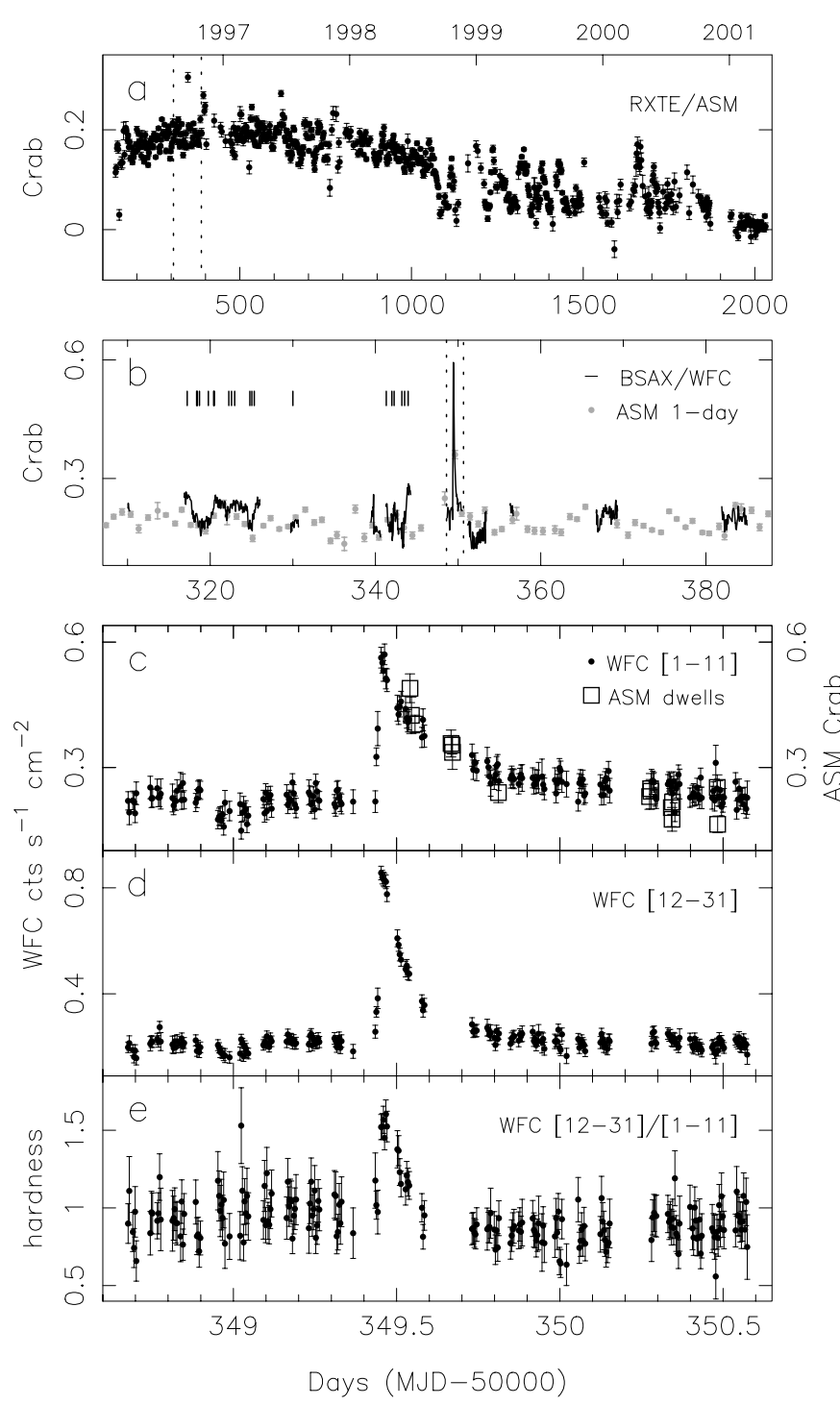

Fig. 1. a) RXTE/ASM (1.5-12 keV) light curve of KS 1731-260 from 1996 January 6 (MJD 50088) to 2001 May 1 (MJD 52030). The data shown are two-day averages and normalized to the count rate of the Crab $\left(75 \mathrm{cts} \mathrm{s}^{-1} \mathrm{SSC}^{-1}\right)$; only intervals with more than one dwell per day were included. b) The BeppoSAX/WFC (2-28 keV) data during the interval 1996 August 12 to November 1 (MJD 50307-50388; marked by the dotted lines in panel a). Data points (typically averages per satellite orbit) which were less than half a day apart are connected to guide the eye, and are normalized to the Crab $\left(2 \mathrm{cts} \mathrm{s}^{-1} \mathrm{~cm}^{-2}\right)$. The gray data points are the one-day averages (with more than one dwell per day) of the RXTE/ASM in the same interval. A long X-ray flare in the WFC data is seen just before MJD 50350 (1996 September 24). The vertical tickmarks indicate the times of the $20 \mathrm{X}$-ray bursts seen with the WFC. They all occur before the flare. $\mathbf{c}-\mathbf{e}$ ) WFC light curves around the flare (marked by dotted lines in panel b) for channels $1-11(2-5 \mathrm{keV} ; \mathbf{c}))$ and $12-31(5-28 \mathrm{keV} ; \mathbf{d}))$ and the hardness ratio of the two above defined energy bands e). The time resolution is $5 \mathrm{~min}$. Overplotted in c) with open squares are the data from individual RXTE/ASM dwells.

in 1996, together with the one-day averages of the ASM. A blow-up of this light curve in two energy bands near the flare is shown in Figs. 1c and d. The flare started near MJD 50349.42 (1996 September 23.42 UT), the observed maximum was seen about 15 min later. Subsequently the source decayed back exponentially to its pre-flare level with a decay time of $\tau_{\exp } \simeq 2.7 \mathrm{hr}(2-28 \mathrm{keV}$; see Table 1$)$. The decay is faster at high energies than at low energies (Table 1). The distinction in the two decay light curves is shown clearly in the hardness curve (Fig. 1e): the spectra harden during the rise to maximum, and soften again during the subsequent decay.

The flare was partly covered by the RXTE/ASM (Fig. 1c) and corresponds to the highest data point in the ASM light curve (Fig. 1a). This enhancement was already noticed by Wijnands (2001), who, however, could not determine its nature because no evidence for softening could be found. We note that there are other high data points which stand out in the ASM light curve (e.g. near MJD 50623: 1997 June 24), which might be remnants of other long X-ray flares. We examined the time-series data mode for each dwell that yielded an anomalously high intensity for KS 1731-260 and found no evidence that a $\mathrm{X}$-ray burst had occurred during any of these observations, which would increase the mean count rate during a dwell. Although some of the high excursions occur when the Sun was close in position in the sky to KS 1731-260 (e.g., near MJD 50400: 1996 November 13), suggesting that the increase is not due to the source itself, we can not rule out that the other high data points were obtained during long $\mathrm{X}$-ray flares (if so, this would indicate recurrence times of less than a year).

In Fig. 2a, we show the count rates $(2-28 \mathrm{keV})$ of the observation near the start of the X-ray flare. The data show a short increase in intensity for about $4 \mathrm{~s}$, about $380 \mathrm{~s}$ after the start of the observation. We will refer to this as "a precursor" hereafter. Afterwards, the intensity decreases somewhat erratically to almost its pre-burst level ${ }^{2}$. Just before the data gap, the flux may start to rise again, but unfortunately we cannot be certain. In Fig. 3a, we again show the count rates, now including the X-ray flare, on a logarithmic time scale. After the gap ( $\simeq 13$ min later), the flux is high and it is possible the flare has already passed its maximum.

We fit the decay light curve of the precursor with an exponential and derive the peak intensity, integrated count

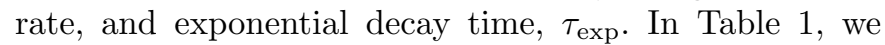
compare these numbers with what is found for the other 63 X-ray bursts seen with the WFC at comparable persistent pre-burst fluxes (i.e. before MJD 51000). We find that the characteristics of the precursor are comparable to the other bursts, but note that the data are not of sufficient quality to determine whether there occurs significant softening during its decay (thus, we cannot prove it is a type I burst). For comparison, we show a light curve around another similar short and weak X-ray burst (one

\footnotetext{
${ }^{2}$ In Figs. 1c and d, the flux appears to increase steadily during the start of the flare. This is due to the choice of data rebinning.
} 


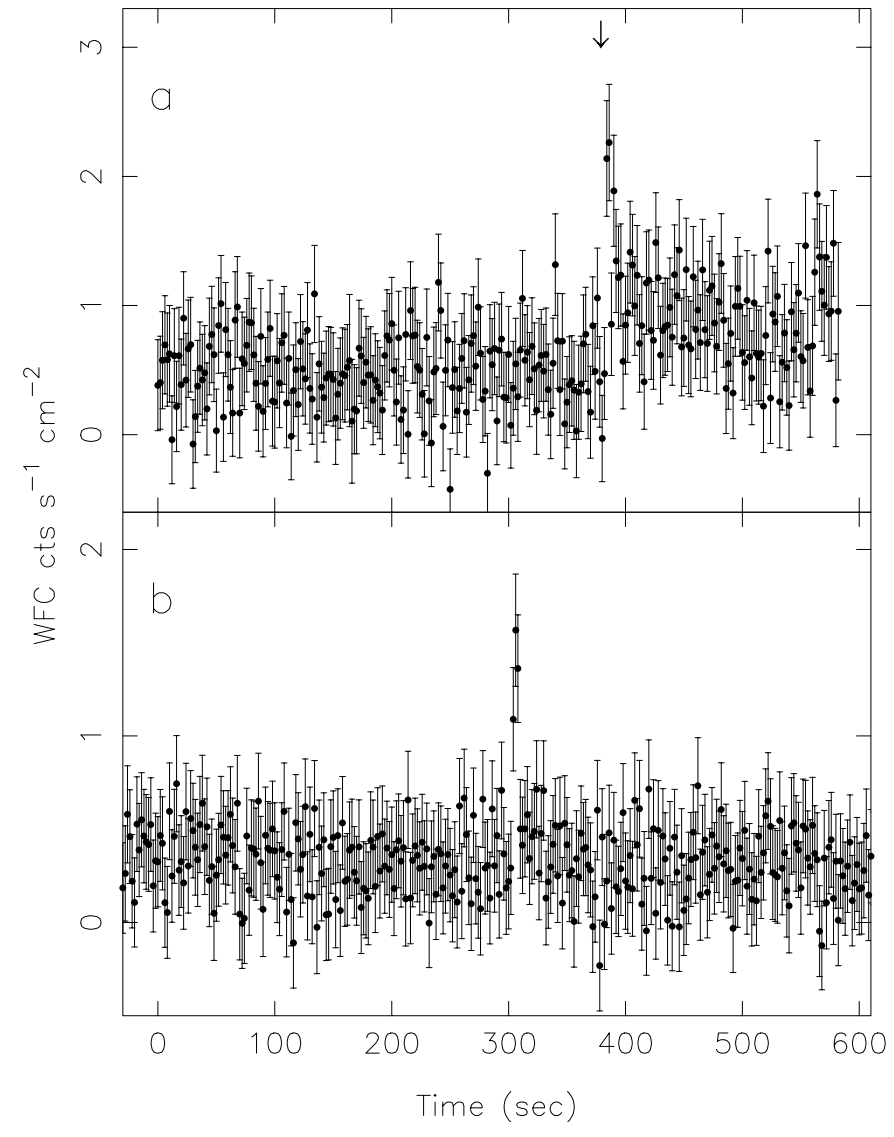

Fig. 2. a) WFC (2-28 keV) count rates of the observation just before the long X-ray flare. Time $=0 \mathrm{~s}$ corresponds to UT 1996 September 23 09:52:02 (MJD 50349.41113); the time resolution is $2 \mathrm{~s}$. Clearly, a short and weak X-ray burst, which we call a precursor, can be seen $380 \mathrm{~s}$ after the start of the observation. The arrow points to the zero time scale of Fig. 3. b) Example of a WFC (2-28 keV) observation around another short and weak X-ray burst. Time $=0 \mathrm{~s}$ corresponds to UT 1997 April 13 19:15:00 (MJD 50551.80208); the time resolution is $2 \mathrm{~s}$.

of the 63 bursts) in Fig. 2b, to illustrate that such bursts are not uncommon in KS 1731-260 (see also Muno et al. 2000).

In Fig. 1b, we show with tickmarks the times of occurrence of normal X-ray bursts observed with the WFC around the time of the long X-ray flare (no bursts were found during the short ASM dwells on the KS 1731-260 region in the same interval). We found 20 normal bursts, all of which occur before the flare. The mean burst rate, corrected for data gaps, is $\sim 3$ per day, but the waiting times between bursts are variable, the shortest being $\simeq 0.08$ day.

No normal bursts were found during the WFC observations which were obtained up to $\simeq 35$ days after the flare, despite considerable exposure time ( $227 \mathrm{ksec})$. Given that 20 bursts occurred during $589 \mathrm{ksec}$ of observation time before the flare we would have expected to find $\simeq 8$ bursts after the flare. We verified the significance of finding no bursts after the flare by performing Monte Carlo simulations. We randomly varied the burst waiting times

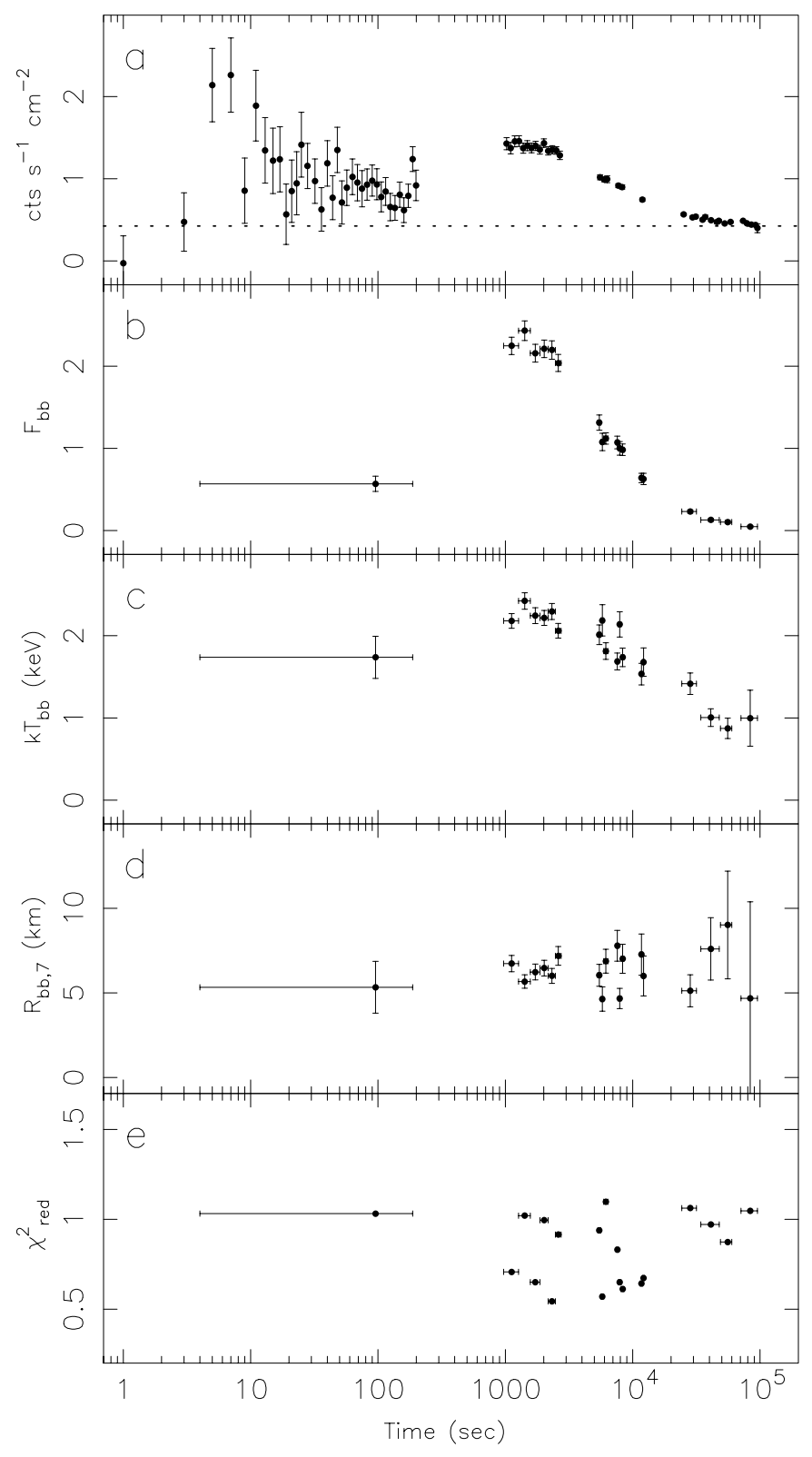

Fig. 3. a) WFC (2-28 keV) count rates of the large flare using a logarithmic time scale, with the data rebinned logarithmically in time. Time $=0 \mathrm{~s}$ corresponds to UT 1996 September 23 09:58:21 (MJD 50349.41552). The dotted line shows the mean count rate before the large X-ray flare. $\mathbf{b}-\mathbf{e}$ ) Results of blackbody fits to the net burst emission (= total burst emission minus pre-burst emission): bolometric black-body flux, $F_{\mathrm{bb}}$, in units of $\left.10^{-8} \mathrm{erg} \mathrm{cm}^{-2} \mathrm{~s}^{-1} \mathbf{b}\right)$, black-body temperature, $k T_{\mathrm{bb}}$ c), apparent black-body radius at $7 \mathrm{kpc}, R_{\mathrm{bb}, 7} \mathrm{~d}$ ) and the goodness of fit expressed in reduced $\chi^{2}$ for 23 degrees of freedom e).

between the lowest observed waiting time (0.08 day) and 0.6 day (i.e. symmetric around 0.34 day). We then determined the number of bursts which occurred within the observing windows after the flare. By doing $10^{6}$ simulations we found that the expected number of bursts is indeed $7.6 \pm 2.4(1 \sigma)$; the probability of observing no bursts after the flare is $1 \times 10^{-4}$. We verified our method by applying 
the same procedure for the interval before the flare: $20 \pm 3.5$ are expected; indeed 20 are seen.

Another way to estimate the significance is to assume that the number of observed normal bursts follows a Poisson distribution. In this case we find that the probability of seeing no bursts instead of the 8 expected is $3.4 \times 10^{-4}$. Finally, in WFC observations during 1997 (first observation was on MJD 50509, i.e. 1997 March 2) and 1998, when the source was at comparable flux levels, the source was also bursting, again with similar burst frequencies as those before the flare (see Fig. 4).

Given the above, we conclude that the burst activity was significantly reduced or had ceased after the long X-ray flare.

\subsection{Spectral behaviour}

In order to derive the characteristics of the long X-ray flare emission, we created spectra throughout its duration, including the precursor. The time intervals were chosen such that we had sufficient statistics per spectrum. The net flare spectra (i.e. total source spectra minus pre-flare persistent emission spectrum $\mathrm{C}$ described below) were satisfactorily fitted with emission from a black-body $\left(\chi_{\text {red }}^{2} \lesssim 1\right.$ for 23 degrees of freedom). We note that some of the $\chi_{\text {red }}^{2}$ values are rather small, perhaps due to a slight overestimate of the systematic errors. As we cannot constrain interstellar absorption well with the WFC, we fixed $N_{\mathrm{H}}$ to the value derived by Barret et al. (2000), $1.3 \times 10^{22}$ atoms $\mathrm{cm}^{-2}$. The results are shown in Figs. 3b-e. The black-body temperature reaches a maximum of $k T=2.4 \pm 0.1 \mathrm{keV}$ and decreases thereafter. The apparant black-body radius at the distance of $7 \mathrm{kpc}$ remains more or less constant throughout the burst with a value of $\simeq 6 \mathrm{~km}$; as expected, the relation between $k T$ and $F_{\mathrm{bb}}$ is consistent with a power law, $F_{\mathrm{bb}} \propto T^{\alpha}$, with $\alpha=3.9 \pm 0.4$. Similar black-body parameters are found for normal X-ray bursts from this source (e.g. Muno et al. 2000).

We also performed spectral fits to the flare spectra not subtracting the pre-flare persistent emission. The fits to the spectra up to $12200 \mathrm{~s}$ into the flare were acceptable: $\chi_{\text {red }}^{2}=0.7-1.2$ (maximum $F_{\mathrm{bb}}=2.9 \pm 0.1 \times$ $10^{-8} \mathrm{erg} \mathrm{cm}^{-2} \mathrm{~s}^{-1}$, maximum $k T=2.2 \pm 0.1 \mathrm{keV}, R_{\mathrm{bb}, 7} \simeq$ $10 \mathrm{~km})$. However, the fits to the spectra of the remainder of the burst were not acceptable: $\chi_{\text {red }}^{2}=2.8-7.7$. This indicates that while it seems more likely the persistent emission was present during the flare, we can not rule out its absence during the first $\sim 3.5 \mathrm{hr}$. In the remainder of the paper we use the result from the spectral fits to the net-flare emission.

We estimated the fluence, $E_{\mathrm{b}}$, of the flare by assuming that the rise from the start to the observed maximum is linear and that the decay after this maximum is described by an exponential. In Table 1, we list the values of $E_{\mathrm{b}}$, the maximum observed net-burst blackbody flux, $F_{\mathrm{bb} \text {,max }}$, the ratio $\tau \equiv E_{\mathrm{b}} / F_{\mathrm{bb} \text {,max }}$, and the ratio $\gamma \equiv F_{\text {pers }} / F_{\text {bb,max }}$ (where $F_{\text {pers }}$ is the bolometric

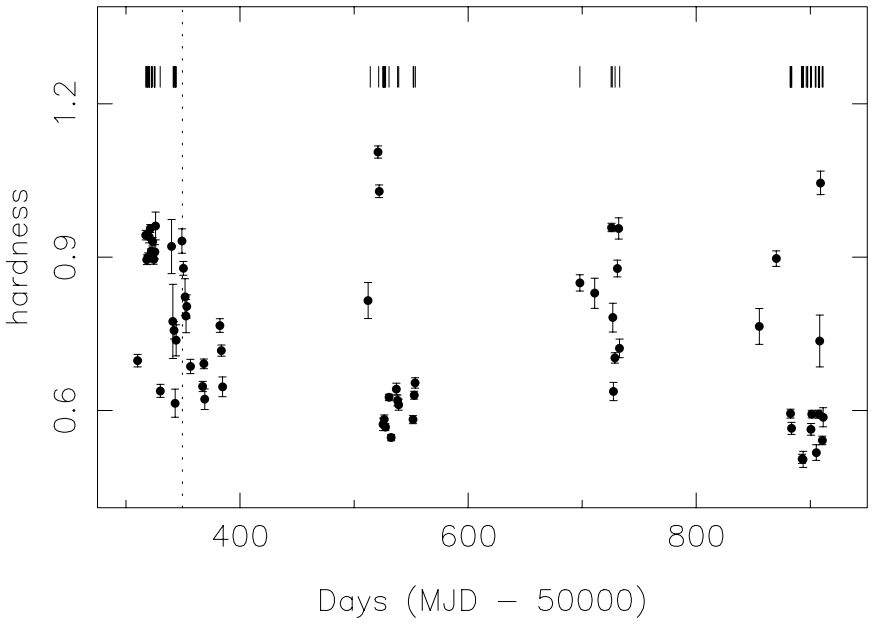

Fig. 4. WFC hardness (as defined in Fig. 1e) curve from MJD 50275-50950 (1996 July 11-1998 May 17) when the persistent X-ray intensity was at comparable levels. Data points are 1-day averages. The dotted line denotes the time of the long X-ray flare. In the upper part we have marked the times of the bursts with bars. Clearly, no bursts are found just after the flare. Normal bursting had resumed in the next campaign.

persistent source flux approximated by extrapolating the observed persistent spectrum between 0.01 and $100 \mathrm{keV}$ ). Note that the values of $\tau$ and $\gamma$ are rather uncertain since we missed the peak of the flare. We find that the fluence $E_{\mathrm{b}}$ of the flare is a factor of 250-425 larger than what is observed for normal X-ray bursts in this source (see e.g. Muno et al. 2000). We note that if we do not use the exponential but simply integrate the observed fluxes during the flare and interpolate over gaps, we obtain a slightly higher fluence of $\simeq 2.4 \times 10^{-4} \mathrm{erg}$. The flare does not reach the Eddington limit during our observations: $F_{\mathrm{bb} \text { max }} \simeq 0.4 L_{\mathrm{Edd}}$ given the Eddington flux of $6.3 \times 10^{-8} \mathrm{erg} \mathrm{cm}^{-2} \mathrm{~s}^{-1}$ inferred by Muno et al. (2000) from radius expansion bursts of KS 1731-260.

\subsection{Persistent emission}

The change in bursting behaviour just after the flare led us to investigate whether the persistent emission is influenced as well. We calculated hardness ratios (as defined in Sect. 4.1) during the interval 1996-1998, when the source was at comparable persistent flux levels. These are shown in Fig. 4; clearly, the source did not have exceptional hardness values after the flare.

In order to quantify the above, we created a total of six X-ray spectra of the persistent emission before and after the flare during different intervals between MJD 50308-50388 (see Table 2). For each spectrum data from various contiguous observations with the same pointing were accumulated. To parametrize the persistent emission we fitted the $2-20 \mathrm{keV}$ spectra to an absorbed cutoff power-law model plus a Gaussian line at $6.4 \mathrm{keV}$ (see Barret et al. 2000). As above, we fixed the value of $N_{\mathrm{H}}$ to $1.3 \times 10^{22}$ atoms $\mathrm{cm}^{-2}$; furthermore, we fixed the line 
Table 1. KS 1731-260 precursor and superburst characteristics.

\begin{tabular}{|c|c|c|}
\hline & Precursor & Type I bursts \\
\hline peak rate ${ }^{a}$ & $2.3 \pm 0.4 \mathrm{cts} \mathrm{s}^{-1} \mathrm{~cm}^{-2}$ & $1.8-5.3(3.6) \mathrm{cts} \mathrm{s}^{-1} \mathrm{~cm}^{-2}$ \\
\hline \multicolumn{3}{|l|}{ integrated } \\
\hline count rate & $\simeq 13 \mathrm{cts} \mathrm{cm}^{-2}$ & $13-45(29) \mathrm{cts} \mathrm{cm}^{-2}$ \\
\hline$\tau_{\exp }(2-28 \mathrm{keV})$ & $5.6_{-1.9}^{+2.8} \mathrm{~s}$ & $2-11(5.5) \mathrm{s}$ \\
\hline \multicolumn{3}{|l|}{ Superburst } \\
\hline parameter & value & $\chi_{\text {red }}^{2} /$ d.o.f. \\
\hline$\tau_{\exp }(2-28 \mathrm{keV})$ & $2.7 \pm 0.1 \mathrm{hr}$ & $0.94 / 116$ \\
\hline$(2-5 \mathrm{keV})$ & $4.4 \pm 0.3 \mathrm{hr}$ & $0.7 / 116$ \\
\hline$(5-28 \mathrm{keV})$ & $2.1 \pm 0.1 \mathrm{hr}$ & $0.8 / 116$ \\
\hline parameter & at earth & at $7 \mathrm{kpc}$ \\
\hline$F_{\text {pers }}^{b}$ & $1.0 \pm 0.1 \times 10^{-8} \mathrm{erg} \mathrm{s}^{-1} \mathrm{~cm}^{-2}$ & $5.9 \pm 0.8 \times 10^{37} \mathrm{erg} \mathrm{s}^{-1}$ \\
\hline$E_{\mathrm{b}}$ & $\simeq 1.7 \times 10^{-4} \mathrm{erg} \mathrm{cm}^{-2}$ & $\simeq 9.8 \times 10^{41} \mathrm{erg}$ \\
\hline$F_{\mathrm{bb}, \max }$ & $2.4 \pm 0.1 \times 10^{-8} \mathrm{erg} \mathrm{s}^{-1} \mathrm{~cm}^{-2}$ & $1.4 \pm 0.1 \times 10^{38} \mathrm{erg} \mathrm{s}^{-1}$ \\
\hline parameter & value & \\
\hline$\tau=E_{\mathrm{b}} / F_{\mathrm{bb}, \max }$ & $\simeq 2.0 \mathrm{hr}$ & \\
\hline$\gamma=F_{\mathrm{pers}} / F_{\mathrm{bb}, \max }$ & $0.41 \pm 0.06$ & \\
\hline
\end{tabular}

Table 2. Persistent emission spectral fit (absorbed cut-off power-law) parameters ${ }^{a}$.

\begin{tabular}{lcccccc}
\hline & $\mathbf{A}$ & $\mathbf{B}$ & $\mathbf{C}$ & $\mathbf{D}$ & $\mathbf{E}$ & $\mathbf{F}$ \\
Interval (MJD-50000) & $318.6-322.2$ & $323.4-326.0$ & $348.7-349.3$ & $350.2-350.5$ & $366.7-369.0$ & $383.1-385.0$ \\
$F_{2-10 \mathrm{keV}}\left(10^{-9} \mathrm{erg} \mathrm{s}^{-1} \mathrm{~cm}^{-2}\right)$ & $4.6 \pm 0.2$ & $4.8 \pm 0.3$ & $5.2 \pm 1.1$ & $5.7 \pm 1.4$ & $4.3 \pm 0.4$ & $4.2 \pm 0.8$ \\
$\Gamma^{b}$ & $1.04 \pm 0.03$ & $1.20 \pm 0.05$ & $1.0 \pm 0.1$ & $1.2 \pm 0.2$ & $1.03 \pm 0.06$ & $0.9 \pm 0.1$ \\
$E_{\text {cut }}(\mathrm{keV})^{c}$ & $4.3 \pm 0.1$ & $4.6 \pm 0.2$ & $5.2 \pm 0.7$ & $5.6 \pm 1.0$ & $4.5 \pm 0.2$ & $3.4 \pm 0.3$ \\
norm. $^{d}$ & $1.34 \pm 0.03$ & $1.63 \pm 0.05$ & $1.3 \pm 0.1$ & $1.5 \pm 0.2$ & $1.17 \pm 0.05$ & $1.26 \pm 0.09$ \\
gnorm. $^{e}\left(\times 10^{-3}\right)$ & $4.1 \pm 0.8$ & $6.6 \pm 1.3$ & $-^{f}$ & $-{ }^{f}$ & $-_{f}^{f}$ & $-^{f}$ \\
$\chi^{2} /$ d.o.f. & $1.6 / 21$ & $0.9 / 21$ & $1.2 / 22$ & $1.1 / 22$ & $1.0 / 22$ & $1.5 / 22$ \\
\hline
\end{tabular}

${ }^{a} N_{\mathrm{H}}$ was fixed to $1.3 \times 10^{22}$ atoms $\mathrm{cm}^{-2}$, see text.

${ }^{b}$ Power-law photon index.

${ }^{c}$ Cut-off energy.

${ }^{d}$ Power-law nomalisation in photons $\mathrm{cm}^{-2} \mathrm{~s}^{-1}$ at $1 \mathrm{keV}$.

e Normalisation in photons $\mathrm{cm}^{-2} \mathrm{~s}^{-1}$ in the line modeled by a Gaussian fixed at $6.4 \mathrm{keV}$ and width $(\sigma)$ of $0.1 \mathrm{keV}$.

${ }^{f}$ Including a Gaussian did not improve the fit significantly.

width of the Gaussian, $\sigma$, to 0.1 . This provided a good description of the spectra ${ }^{3}$; the results are presented in Table 2. The unabsorbed persistent luminosity before and after the flare was $\simeq 0.1 L_{\mathrm{Edd}}(2-10 \mathrm{keV})$.

We conclude that the persistent $\mathrm{X}$-ray spectra days to weeks after the flare (when no normal bursts occurred) are not different from the spectra days to weeks before

\footnotetext{
${ }^{3}$ Previously, a black-body component (or a disk black-body component) has been included in spectral fits (Barret et al. 2000; Narita et al. 2001). We, however, found their inclusion made the fits rather unstable, while providing little (spectra A and $\mathrm{F}$ ) or no (B-E) improvement.
}

and months to years after the flare (when normal bursts did occur).

\section{Discussion}

\subsection{Very long thermonuclear runaway events}

In many of its characteristics, the long X-ray flare resembles type I bursts from KS 1731-260 (e.g. Muno et al. 2000) as well as type I bursts from other sources. The rise to maximum is relatively fast with respect to the decay, the decay is exponential, and the spectra soften (indicating cooling) during the decay. This strongly 
Table 3. Properties of superbursts ordered along their exponential decay time ${ }^{a}$.

\begin{tabular}{|c|c|c|c|c|c|c|}
\hline source & $4 U 1820-30$ & Ser X-1 & $4 \mathrm{U} 1735-44$ & $4 U 1636-53$ & GX $3+1$ & KS $1731-260$ \\
\hline instrument & PCA & WFC & WFC & ASM,PCA & ASM & WFC,ASM \\
\hline energy range & $2-60 \mathrm{keV}$ & $2-28 \mathrm{keV}$ & $2-28 \mathrm{keV}$ & $1.5-12 \mathrm{keV}$ & $1.5-12 \mathrm{keV}$ & $2-28 \mathrm{keV}$ \\
\hline duration (hr) & $>2.5$ & $\sim 4$ & $\sim 7$ & $\gtrsim 1-3$ & $>4.4$ & $\sim 12$ \\
\hline precursor? & yes & $?$ & $?$ & $?$ & $?$ & yes \\
\hline$\tau_{\exp }(\mathrm{hr})$ & $\simeq 1$ & $1.2 \pm 0.1$ & $1.4 \pm 0.1$ & $1.5 \pm 0.1 / 3.1 \pm 0.5$ & $1.6 \pm 0.2$ & $2.7 \pm 0.1$ \\
\hline$L_{\text {pers }}\left(L_{\mathrm{Edd}}\right)^{b}$ & $\simeq 0.1$ & $\simeq 0.2$ & $\simeq 0.25$ & $\sim 0.1$ & $\sim 0.2$ & $\simeq 0.1$ \\
\hline$k T_{\max }(\mathrm{keV})$ & $\simeq 3.0$ & $2.6 \pm 0.2$ & $2.6 \pm 0.2$ & $?$ & $\sim 2$ & $2.4 \pm 0.1$ \\
\hline$L_{\text {peak }}\left(10^{38} \mathrm{erg} \mathrm{s}\right)^{c, d}$ & $3.4 \pm 0.1$ & $1.6 \pm 0.2$ & $1.5 \pm 0.1$ & $\sim 1.2$ & $\sim 0.8$ & $1.4 \pm 0.1$ \\
\hline$E_{\mathrm{b}}\left(10^{42} \mathrm{erg}\right)$ & $\gtrsim 1.4$ & $\simeq 0.8$ & $\gtrsim 0.5$ & $\sim 0.5-1$ & $\gtrsim 0.5$ & $\simeq 1.0$ \\
\hline$\tau \equiv E_{\mathrm{b}} / L_{\text {peak }}(\mathrm{hr})^{d}$ & $\gtrsim 1.1$ & $\simeq 1.4$ & $\gtrsim 0.9$ & $\sim 1.2-2.3$ & $\gtrsim 1.5$ & $\simeq 2.0$ \\
\hline$\gamma \equiv L_{\mathrm{pers}} / L_{\mathrm{peak}}^{d}$ & $\simeq 0.1$ & $\simeq 0.4$ & $\sim 0.4$ & $\sim 0.2$ & $\sim 0.5$ & $\simeq 0.4$ \\
\hline$t_{\text {no bursts }}(\text { days })^{e}$ & $?$ & $\sim 34$ & $>7.5$ & $?$ & $?$ & $>35$ \\
\hline $\mathrm{H} / \mathrm{He}$ or $\mathrm{He}$ donor & $\mathrm{He}$ & $?$ & $\mathrm{H} / \mathrm{He}$ & $\mathrm{H} / \mathrm{He}$ & $?$ & $?$ \\
\hline references $^{f}$ & S00, SB01 & $\mathrm{C} 01$ & $\mathrm{C} 00$ & W01 & K01 & this paper \\
\hline
\end{tabular}

${ }^{a}$ A question mark denotes an unknown value.

${ }^{b}$ We used the $0.01-100 \mathrm{keV}$ unabsorbed flux from spectral fits and the observed maximum flux during radius expansion bursts.

${ }^{c}$ Unabsorbed bolometric peak (black-body) luminosity.

${ }^{d}$ Since the rise to maximum was only observed for $4 \mathrm{U} 1820-30$, the values for the other sources are to be used with caution.

$e$ Time of cessation of normal X-ray bursts after the superburst.

${ }^{f} \mathrm{C} 00=$ Cornelisse et al. (2000), C01 = Cornelisse et al. (2001), K01 = Kuulkers (2001a,b), S00 = Strohmayer (2000),

S01 = Strohmayer \& Brown (2001), W01 = Wijnands (2001).

suggests a thermo-nuclear runaway event on the neutron star. The duration of the X-ray flare, however, is about half a day, i.e., a factor of $\sim 1500-4000$ longer than normal X-ray bursts from this source. Therefore, the fluence, $\simeq 10^{42} \mathrm{erg}$ (at $7 \mathrm{kpc}$ ), is also much larger than typical. The longest "normal" X-ray bursts seen in general are much shorter and less energetic, and all are radius expansion events: they had durations on the order of half an hour (Hoffman et al. 1987: probably from 4U 1708-23; Kuulkers et al. 2001: GX $17+2$ ), fluences of $\simeq 10^{41} \mathrm{erg}$, exponential decay times of $5.5 \mathrm{~min}(4 \mathrm{U} 1708-23)$ and $4.6 \mathrm{~min}$ (GX 17+2), and ratios of the fluence to peak netburst flux $(\tau)$ of $\simeq 5.4 \mathrm{~min}$ and $6.6 \mathrm{~min}$, respectively. These values are an order of magnitude smaller than those for the superbursts.

The long X-ray flare resembles the superbursts seen in five other sources (4U 1820-30, Ser X-1, 4U 1735-44, $4 \mathrm{U} 1636-53$ and GX $3+1$ ), as can be seen in Table 3, where we compare the properties of all seven flares observed to date. The duration of the superbursts are estimated by eye from the light curves and the hardness curves. With respect to duration of about half a day the flare in KS 1731-260 is the longest among the superbursts seen so far.

The superburst seen in 4U 1820-30 (Strohmayer 2000; Strohmayer \& Brown 2001) is the only one for which the full rise to maximum has been observed. That superburst was immediately preceeded by a normal X-ray burst. This "precursor" lasted for $\simeq 20 \mathrm{~s}$. Both the "precursor" and the superburst exhibited radius expansion, indicating the luminosity reached the Eddington limit. The superbursts from all sources but $4 \mathrm{U} 1820-30$ seem to be a factor $2^{-}$ 4 fainter than their brightest normal bursts, although we should stress again that only in $4 \mathrm{U} 1820-30$ the rise to maximum was fully covered. Next best is KS 1731-260, for which we observed the start and found a short $(\simeq 4 \mathrm{~s})$ and weak burst, most probably a type I burst, preceding the superburst by $\gtrsim 200 \mathrm{~s}$. Note that the precursor does not necessarily need to be a trigger of the superburst (see also Strohmayer \& Brown 2001). The observation of precursors close to the superbursts is another difference from normal (type I) bursts, which do not have such precursors (see e.g. Lewin et al. 1993).

We found evidence that the occurrence of normal bursts stopped for more than a month after the superburst in KS 1731-260. Similarly, no bursts were observed for about a week after the superburst in $4 \mathrm{U}$ 1735-44, whereas in Ser X-1 no bursts were seen for $\sim 5$ weeks after the superburst (Cornelisse et al. 2000, 2001; see also Table 3). This suggests that the superburst has profound influence on the outer envelope of the neutron star where normal bursts originate. The outer envelope may be heated by the energy generated by superburst, causing the burning of hydrogen and/or helium to become stable (see Cumming \& Bildsten 2001). However, the persistent emission of KS 1731-260 during the non-bursting interval is not significantly different compared to intervals of normal bursting. This seems evidence against the idea that the 
neutron star is significantly hotter, but might also just be because the observed persistent emission is dominated by emission from the accretion disk.

\subsection{Unstable carbon burning...}

Assuming unstable nuclear processes, we can use the time scales and total energy to estimate where the burning occurs, how much matter needs to be involved, etc. (see also Cumming \& Bildsten 2001; Strohmayer \& Brown 2001). Since the fusion of elements higher than hydrogen releases about $1 \mathrm{MeV}$ per nucleon ( $\simeq 10^{18} \mathrm{erg} \mathrm{g}^{-1}$; see, e.g., Lamb \& Lamb 1978), one needs to process roughly $10^{24} \mathrm{~g}$ of matter to produce the total energy of $10^{42} \mathrm{erg}$ released during a superburst. With the mass accretion rates of $\sim 10^{-9} M_{\odot} \mathrm{yr}^{-1}$ (as appropriate for sources which radiate at $\left.\sim 0.1-0.3 L_{\mathrm{Edd}}\right)$ the expected recurrence time is at least half a year (depending on how much of the accreted matter is consumed during a superburst). This indicates that they are indeed rare events.

The above mass estimate corresponds to an ignition column depth $y=\Delta M / 4 \pi R_{\mathrm{ns}}^{2} \simeq 8 \times 10^{10} \mathrm{~g} \mathrm{~cm}^{-2}$ (where $\Delta M$ is the amount of burned matter and $R_{\mathrm{ns}}$ the radius of the neutron star; we take $R_{\mathrm{ns}}=10 \mathrm{~km}$ ). At these column depths, the thermal time is on the order of $1.5-3 \mathrm{hrs}$, almost independent of the temperature (see, e.g., Brown \& Bildsten 1998). This is in excellent agreement with the decay time scale of the superbursts. We note that the density at column depths $\sim 8 \times 10^{10} \mathrm{~g} \mathrm{~cm}^{-2}$ is $\rho \simeq 10^{8} \mathrm{~g} \mathrm{~cm}^{-3}$ (see, e.g., Brown \& Bildsten 1998).

The above arguments have led to the suggestion that superbursts are due to unstable carbon burning deeper in the neutron star, in a layer beneath the (un)stable hydrogen/helium or helium layer (Cumming \& Bildsten 2001; Strohmayer \& Brown 2001). Obviously, in order for this to work, the ashes of the burning hydrogen/helium layer need to contain carbon. One way to achieve this is to have stable burning of helium. This may occur in 4U 1820-30, in which the neutron star is thought to accrete pure helium because the companion is most likely a helium white dwarf (based on the very short orbital period, Rappaport et al. 1987), which is consistent with the short bursts this source shows. 4U1820-30 shows long intervals in which no normal X-ray bursts occur, which gave ample time to generate the necessary amount of carbon (Strohmayer \& Brown 2001).

The above mechanism cannot work for all systems, however. In particular, for $4 \mathrm{U} 1735-44$ and $4 \mathrm{U}$ 1636-53 it has been shown that the donors provide hydrogen-rich material (Augusteijn et al. 1998). For burning of a hydrogen/helium mix, one expects only small amounts of carbon to be produced, both for the case that the burning is stable and for the case that it is unstable (e.g. Schatz et al. 1999, 2001). In particular, during normal bursts temperatures in excess of $\sim 10^{9} \mathrm{~K}$ are reached and a break-out of the CNO cycle occurs, creating elements far beyond the iron group. Nevertheless, over a sufficiently long time (many years for mass accretion rates of a few tenth of Eddington) sufficient carbon may be produced to cause a superburst (Cumming \& Bildsten 2001).

Another issue is whether the burning of pure carbon can be unstable at such low column depths as $8 \times$ $10^{10} \mathrm{~g} \mathrm{~cm}^{-2}$. Brown \& Bildsten (1998) found ignition at $>5 \times 10^{12} \mathrm{~g} \mathrm{~cm}^{-2}$ for pure carbon at Eddington accretion rates. The superbursters accrete near a tenth of the Eddington accretion rate, making it even more of a challenge to ignite carbon at the lower pressures implied by the observations. Strohmayer \& Brown (2001) show that one may underestimate the amount of energy released when integrating the superburst X-ray flux, and therefore also the amount of carbon consumed during the superburst. According to the authors, most of the energy is released as neutrinos, and most of the heat produced by the burning largely flows inwards. If so, the large amounts of carbon required ( $\left.\gtrsim 10^{26} \mathrm{~g}\right)$, imply very long recurrence times for the superbursts in $4 \mathrm{U} 1820-40$ (decades). An alternative suggestion is that the precursor helium flash triggered unstable carbon burning, but Strohmayer \& Brown (2001) consider this to be less likely.

Cumming \& Bildsten (2001) have a different view. They point out that the heavy elements in the ashes will reduce the thermal conductivity, which may lead to large temperature gradients and ignition at the observed column depth. They find this works for mass accretion rates above $10 \%$ of the Eddington rate and for mass fractions of carbon larger than 0.1. They argue that the fact that up to now superbursts have only been reported in systems with accretion rates between 10 and $30 \%$ of Eddington, is that at lower mass accretion rates the superbursts are easier to distinguish from the persistent emission.

Cumming \& Bildsten (2001; see also Strohmayer \& Brown 2001) argued that the cooling following the carbon burning may provide a large heat flux into the hydrogen/helium burning layers, stabilizing the burning there. For mass accretion rates of 10-20\% of Eddington bursting would then cease for about 15-20 days after the superburst. This is more or less consistent with what is observed in KS 1731-260 and Ser X-1.

\section{3. ... or runaway electron capture?}

Although with unstable carbon burning it appears possible to understand the superbursts, there may be an interesting alternative. The densities at which the Fermi energy of the electrons, which provide the pressure in the outer layers, becomes equal to the difference in restmass of a neutron and a proton are $2-3 \times 10^{7} \mathrm{~g} \mathrm{~cm}^{-3}$ (where the exact number depends on the composition). This density corresponds to a column density of $y \simeq$ $2 \times 10^{10} g_{14.3}^{-1} \mathrm{~g} \mathrm{~cm}^{-2}$, where $g_{14.3}$ is the surface gravity normalised to $10^{14.3} \mathrm{~cm} \mathrm{~s}^{-2}$, appropriate for a neutron star mass $M_{\mathrm{ns}}=1.4 M_{\odot}$ and $R_{\mathrm{ns}}=10 \mathrm{~km}$ (e.g. Bildsten \& Cumming 1998). This is not too different from the values inferred above. Hence, if any protons are present, they will 
capture electrons via the $p\left(\mathrm{e}^{-}, \nu\right) n$ reaction (Rosenbluth et al. 1973; see also Taam et al. 1996; Bildsten \& Cumming 1998). The released neutrons are then captured by heavy nuclei (rather than by protons, see also below) releasing $\simeq 7-8 \mathrm{MeV}$ per nucleon (Bildsten \& Cumming 1998). Depending on the temperature (which sets the precise distribution of electron energies) and the thermal conductivity, this process may be unstable: an increase in temperature leads to a larger fraction of the electrons at high energies; if the region does not cool sufficiently fast, this leads to more captures, a further increase in temperature, etc.

There have been some studies of the above described mechanism, but only for the case in which no heavy nuclei are present and the neutrons are captured by protons. This process is called "deep hydrogen burning" (Hameury et al. 1982, 1983; Woosley \& Weaver 1984; Fushiki et al. 1992; Taam et al. 1993, 1996). This type of burning can become unstable when the neutron star envelope has a low temperature $\left(\$ 10^{7} \mathrm{~K}\right)$ and/or when the abundance of CNO elements is very low $(Z \lesssim 0.001)$. The latter may not be as unrealistic as it appears at first, since the original CNO abundance received from the donor may be significantly reduced due to nuclear spallation processes in the accretion shock (Bildsten et al. 1992). In the models, long ( $>30 \mathrm{~min})$ X-ray bursts have been produced at moderate accretion rates $\left(\sim 0.1 \dot{M}_{\text {Edd }}\right)$, with total nuclear energy releases of up to several times $10^{41} \mathrm{erg}$ (Woosley \& Weaver 1984, their model 6; Fushiki et al. 1992). This is still an order of magnitude less than observed in superbursts (but rather similar to the "long" X-ray bursts seen from $4 \mathrm{U} 1708-23$ and GX $17+2$ !).

Several other questions have to be answered as well, however. Foremost, whether there is (enough) hydrogen left after the (un)stable hydrogen/helium burning. Various calculations suggest residual hydrogen mass fractions of $X_{\mathrm{H}} \simeq 0.04-0.2$ for mass accretion rates of $>10^{-9} M_{\odot} \mathrm{yr}^{-1}$ (e.g. Taam et al. 1996; see also Koike et al. 1999). (Of course, this mechanism cannot explain the superburst in $4 \mathrm{U} 1820-30$, since for that source there are no protons to begin with. We note, however, that this superburst seems to be somewhat different from the others, so a different mechanism may not be unexpected.)

To produce $10^{42} \mathrm{erg}$ one needs $\simeq 10^{23} \mathrm{~g}$ of residual hydrogen (given the energy release of $7-8 \mathrm{MeV}$ per nucleon). Thus a total accreted matter of $5-25 \times 10^{23} \mathrm{~g}$ is needed; for accretion rates of $\sim 10^{-9} M_{\odot} \mathrm{yr}^{-1}$ the recurrence times of superbursts would then be $0.25-1.25 \mathrm{yr}$. This is an order of magnitude shorter than the case for unstable carbon burning as estimated by Cumming \& Bildsten (2001). We estimate the maximum expected energy release for runaway electron capture from $E_{\max }=4 \pi R_{\mathrm{ns}}^{2} y E_{\mathrm{nuc}} X_{\mathrm{H}}=$ $2 \times 10^{41} R_{\mathrm{ns}, 10}^{4} X_{\mathrm{H}, 0.1} \mathrm{erg}$, where $R_{\mathrm{ns}}=10 R_{\mathrm{ns}, 10} \mathrm{~km}$ is the radius of the neutron star, $E_{\text {nuc }}$ the above quoted energy release, and $X_{\mathrm{H}}=0.1 X_{\mathrm{H}, 0.1}$ (the fourth-power dependence on radius arises because of the dependence of $y$ on the surface gravity). This may be consistent with the observed value of $10^{42} \mathrm{erg}$, if the radius of the neutron star is larger than $10 \mathrm{~km}$, as is indeed inferred for many equations of state (see, e.g., the review by Lattimer \& Prakash 2000 ), and/or the residual mass fraction of hydrogen is larger than 0.1. Thus, unstable electron capture appears a viable alternative mechanism for the superbursts.

Acknowledgements. We thank Lars Bildsten, Andrew Cumming and Ron Taam for discussions, in particular about the possibility of the superbursts being due to electron capture. The BeppoSAX satellite is a joint Italian and Dutch programme. We made use of quick-look results provided by the ASM/RXTE team. MHvK acknowledges support from the Royal Netherlands Academy of Science KNAW.

\section{References}

Augusteijn, T., van der Hooft, F., de Jong, J. A., van Kerkwijk, M. H., \& van Paradijs, J. 1998, A\&A, 332, 561

Barret, D., Olive, J. F., Boirin, L., et al. 2000, ApJ, 533, 329

Bildsten, L., Salpeter, E. E., \& Wasserman, I. 1992, ApJ, 384, 183

Bildtsen, L., \& Cumming, A. 1998, ApJ, 506, 842

Boella, G., Butler, R. C., Perola, G. C., et al. 1997, A\&AS, 122, 299

Brown, E. F. 2000, ApJ, 531, 988

Brown, E. F., \& Bildsten, L. 1998, ApJ, 496, 915

Cornelisse, R., Heise, J., Kuulkers, E., Verbunt, F., \& in 't Zand, J. J. M. 2000, A\&A, 357, L21

Cornelisse, R., Kuulkers, E., in 't Zand, J. J. M., Verbunt, F., \& Heise, J. 2001, A\&A, in press [astro-ph/0111263]

Cumming, A., \& Bildsten, L. 2001, ApJ, 559, L127

Fushiki, I., Taam, R. E., Woosley, S. E., \& Lamb, D. Q. 1992, ApJ, 390, 634

Hameury, J. M., Bonazzola, S., Heyvaerts, J., \& Ventura, J. 1982, A\&A, 111, 242

Hameury, J. M., Heyvaerts, J., \& Bonazzola, S. 1983, A\&A, 121,259

Hoffman, J. A., Lewin, W. H. G., Doty, J., et al. 1978, ApJ, 221, L57

Jager, R., Mels, W. A., Brinkman, A. C., et al. 1997, A\&AS, 125,557

Koike, O., Hashimoto, M., Arai, K., \& Wanajo, S. 1999, A\&A, 342,464

Kuulkers, E. 2001a, ATel \#68

Kuulkers, E. 2001b, A\&AL, submitted

Kuulkers, E., in 't Zand, J. J. M., Cornelisse, R., et al. 2000, A\&A, 358, 993

Kuulkers, E., Homan, J., van der Klis, M., Lewin, W. H. G., \& Méndez, M. 2001, A\&A, in press [astro-ph/0105386]

Lamb, D. Q., \& Lamb, F. K. 1978, ApJ, 220, 291

Lattimer, J. M., \& Prakash, M. 2001, ApJ, 550, 426

Lewin, W. H. G., Vacca, W. D., \& Basinska, E. M. 1984, ApJ, 277, L57

Lewin, W. H. G., van Paradijs, J., \& Taam, R. E. 1993, Space Sci. Rev., 62, 223

Muno, M. P., Fox, D. W., Morgan, E. H., \& Bildsten, L. 2000, ApJ, 542, 1016

Narita, T., Grindlay, J. E., \& Barret, D. 2001, ApJ, 547, 420

Rappaport, S., Ma, C. P., Joss, P. C., \& Nelson, L. A. 1987, ApJ, 322, 842 
Rosenbluth, M. N., Ruderman, M., Dyson, F., et al. 1973, ApJ, 184,907

Revnivtsev, M. G., \& Sunyaev, R. A. 2001, A\&AL, submitted [astro-ph/0108323]

Revnivtsev, M. G., \& Sunyaev, R. A. 2002, Astron. Lett., in press [astro-ph/0108120]

Schatz, H., Bildsten, L., Cumming, A., \& Wiescher, M. 1999, ApJ, 524, 1014

Schatz, H., Aprahamian, A., Barnard, V., et al. 2001, Phys. Rev. Lett., 86, 3471

Smith, D. A., Morgan, E. H., \& Bradt, H. 1997, ApJ, 479, L137

Smith, D. A., Levine, A., Bradt, H., et al. 2001, ApJS, in press [astro-ph/0103357]

Strohmayer, T. E. 2000, HEAD, 32, 24.10

Strohmayer, T. E., \& Brown, E. F. 2001, ApJ, in press [astro-ph/0108420]
Sunyaev, R. A., \& the Kvant Team 1989, IAU Circ., 4839

Sunyaev, R. A., Gilfanov, M., Churazov, E., et al. 1990, Sov. Astron. Lett., 16, 59

Taam, R. E., Woosley, S. E., Weaver, T. A., \& Lamb, D. Q. 1993, ApJ, 413, 324

Taam, R. E., Woosley, S. E., \& Lamb, D. Q. 1996, ApJ, 459, 271

van Paradijs, J., Penninx, W., \& Lewin W. H. G. 1988, MNRAS, 233, 437

Wijnands, R. 2001, ApJ, 554, L59

Wijnands, R., Miller, J. M., Markwardt, C., Lewin, W. H. G., \& van der Klis, M. 2001a, ApJ, 560, L159

Wijnands, R., Groot, P. J., Miller, J. L., et al. 2001b, ATel \#72

Wijnands, R. A. D., \& van der Klis, M. 1997, ApJ, 482, L65

Woosley, S. E., \& Weaver, T. A. 1984, in High Energy Transients in Astrophysics, AIP, 275 